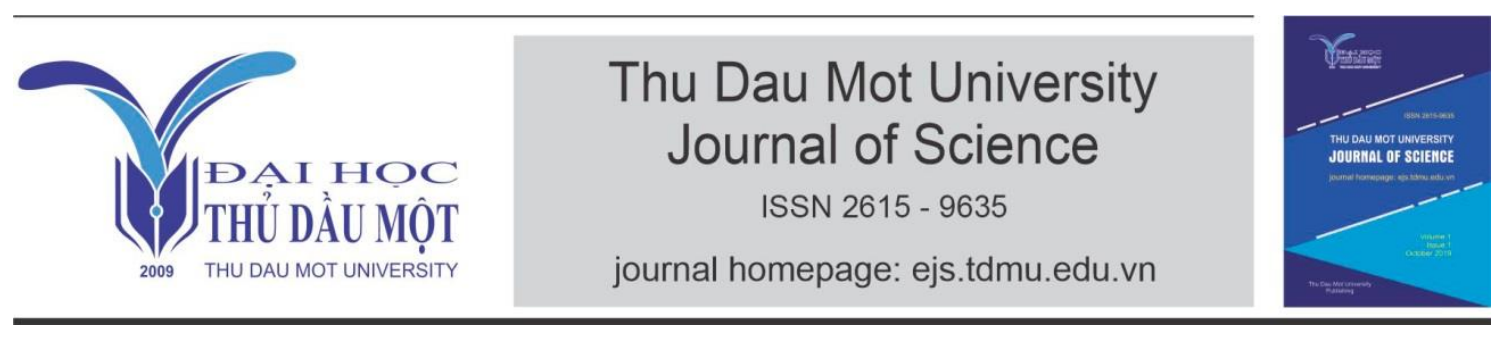

\title{
Distribution system reconfiguration using water cycle algorithm
} by Tran Van Thanh (Thu Dau Mot University)

Article Info: Received May $7^{\text {th }}, 2021$, Accepted Nov. $25^{\text {th }}, 2021$, Available online Dec. $15^{\text {th }}, 2021$ Corresponding author: thanhtv@tdmu.edu.vn https://doi.org/10.37550/tdmu.EJS/2021.04.259

\begin{abstract}
High power losses are a great concern in operating electric distribution system. Reconfiguration is one of the most economic approaches for reducing power losses of the system. This study suggests a technique for dealing with the distribution system reconfiguration problem based on a water cycle algorithm for minimizing active power loss. The water cycle algorithm is a recently developed metaheuristic algorithm that inspired the process of water circulation for solving optimization problems. The effectiveness and performance of the water cycle algorithm were tested on the 33-node and 69-node systems. The water cycle algorithm was applied to determine the best configuration of the distribution system for active power loss minimization. The results yielded by the water cycle algorithm were compared with other optimization algorithms in the literature and the comparisons showed that the water cycle algorithm obtained good quality of solution for the problem. Therefore, the water cycle algorithm is the potential method for the distribution system reconfiguration problem.
\end{abstract}

Keywords: distribution system reconfiguration, electric distribution system, optimization, water cycle algorithm

\section{Introduction}

Electric distribution system (EDS) transmits electricity from the transmission system to individual consumers. In recent, EDS is becoming large and complex leading to higher losses of system. Hence, reduction of power losses has constituted one of the most important objectives in operating EDS. Among techniques of reducing power loss, distribution system reconfiguration (DSR) is one of the most economic techniques among them. It is implemented by changing the status of opened and closed switches in 
EDS (Biswas, Mallipeddi, Suganthan, \& Amaratunga, 2017).

In the last two decades, the DSR problem has been studied by many modern methods such as genetic algorithm (GA), particle swarm optimization (PSO), fireworks algorithm (FWA), cuckoo search algorithm (CSA), harmony search algorithm (HSA), and bacterial foraging optimization (BFO). In (Zhu, 2002), the GA was applied to deal with the DSR problem to reduce power loss. In (Abdelaziz, Mohammed, Mekhamer, \& Badr, 2009; Othman, El-Fergany, \& Abdelaziz, 2015), PSO was used to handle the DSR problem for power loss minimization. In (Mohamed Imran \& Kowsalya, 2014; Mohamed Imran, Kowsalya, \& Kothari, 2014), FWA was suggested for the DSR problem to improve the node voltage and reduce power loss. In (Nguyen \& Nguyen, 2019; Nguyen \& Truong, 2015; Nguyen, Truong, \& Phung, 2016), CSA was successful applied to the DSR problem for improving node voltage and reducing power loss. In (Srinivasa Rao, Narasimham, Ramalinga Raju, \& Srinivasa Rao, 2011), HSA was used to find the optimal configuration for power loss reduction. In (Naveen, Sathish Kumar, \& Rajalakshmi, 2015), BFO was solved the DSR problem to reduce power loss. Many optimization methods have been developed in recent years. Hence, the study of applying new methods to the DSR problem is a matter of great concern to contribute more effective methods in solving the DSR problem.

The water cycle algorithm (WCA) developed by (Eskandar, Sadollah, Bahreininejad, \& Hamdi, 2012) is a recent metaheuristic algorithm for dealing with optimization problems. The basic concept of WCA is based on the circulation of water in nature, including the movement of rivers into the sea. In this study, the WCA was proposed for solving the DSR problem for power loss minimization in EDS. The effectiveness of the proposed WCA was tested on 33-node and 69-node systems and the obtained results were compared to those from other methods available in the literature.

In the rest of the paper, Section 2 includes problem formulation for the DSR problem. Section 3 explains the application of WCA to the DSR problem. Section 4 includes simulation results. Lastly, the conclusion is given in section 5.

\section{Problem Formulation}

The objective of the DSR problem is to minimize active power loss $\left(P_{L}\right)$ through the optimal setting of opened switches while fulfilling the operational constraints of an EDS. Hence, the DSR problem is generally defined as follows:

$$
O F=\operatorname{Min}\left(P_{L}\right)=\operatorname{Min}\left(\sum_{k=1}^{N_{L}} R_{k} I_{k}^{2}\right)
$$

where $R_{k}$ is the resistance of the $k^{\text {th }}$ branch, $I_{k}$ is the current passing through that branch, and $N_{L}$ is the number of branches in a radial distribution system. 
The operational constraints for the DSR problem are given as follows:

i. Real and reactive power balance:

$$
\begin{gathered}
P_{S S}=\sum_{j=1}^{N_{B}} P_{D, j}+\sum_{k=1}^{N_{L}} P_{L, k} \\
Q_{S S}=\sum_{j=1}^{N_{B}} Q_{D, j}+\sum_{k=1}^{N_{L}} Q_{L, k}
\end{gathered}
$$

Where $P_{S S}$ and $Q_{S S}$ are the real and reactive powers supplied from the slack bus, respectively; $P_{D, j}$ and $Q_{D, j}$ are the real and reactive power of load demands at the $j^{\text {th }}$ bus, respectively; $P_{L, k}$ and $Q_{L, k}$ are the real and reactive power losses in the $k^{\text {th }}$ branch, respectively; $N_{B}$ is the total number of buses in a distribution system.

ii. Voltage constraints:

$$
V_{\min , i} \leq V_{i} \leq V_{\max , i} ; \quad i=1, \ldots, N_{B}
$$

where $V_{\min , i}$ and $V_{\max , i}$ are the voltage limits at the $i^{\text {th }}$ bus.

iii. Thermal constraints:

$$
\left|I_{k}\right| \leq\left|I_{\text {max }, k}\right| ; \quad k=1, \ldots, N_{L}
$$

where $I_{\max , k}$ is the maximum current allowed to flow through the $k^{\text {th }}$ branch.

iv. Radial configuration constraint: the EDS must ensure the radial configuration and serve all loads as follows (Abdelaziz, Mohamed, Mekhamer, \& Badr, 2010; Abdelaziz et al., 2009):

$$
\operatorname{det}(A)=\left\{\begin{array}{cc}
1 \text { or }-1 & (\text { radial system }) \\
0 & (\text { not radial })
\end{array}\right.
$$

where $A$ is a branch-bus incidence matrix in an EDS.

\section{Methodology}

The Water Cycle Algorithm (WCA) was developed on the basis of observation of the water circulation procedure and the flow process of streams and rivers to the sea. Further details of MOWCA can be found in (Eskandar et al., 2012). To implement the WCA to DSR problem, each individual of the initial population representing the design variables are defined as follows:

$$
X_{i}=\left[S W_{1}, \ldots, S W_{N_{S W}}\right], \quad i=1, \ldots, N_{p o p}
$$

where $S W_{j}$ is the positions of opened switches in EDS, $N_{S W}$ is the number of opened switches.

Implementation of WCA for the DSR problem is stated as follows: 
Step 1: Set initial parameters of WCA, including ( $N_{\text {pop }}, N_{s r}, d_{\max }$, and MaxIteration).

Step 2: Create a random initial population of raindrops as follows:

$$
\text { Total Population }=\left[\begin{array}{c}
\text { Sea } \\
\text { River }_{1} \\
\text { River }_{2} \\
\text { River }_{3} \\
\vdots \\
\text { Stream }_{N_{s+1}} \\
\text { Stream }_{N_{s+2}} \\
\text { Stream }_{N_{s+3}} \\
\vdots \\
\text { Stream }_{N_{\text {pop }}}
\end{array}\right]=\left[\begin{array}{cccc}
x_{1}^{1} & x_{2}^{1} & \ldots & x_{N}^{1} \\
x_{1}^{2} & x_{2}^{2} & \ldots & x_{N}^{2} \\
\vdots & \vdots & \vdots & \vdots \\
x_{1}^{N_{p o p}} & x_{2}^{N_{p o p}} & \ldots & x_{N}^{N_{p o p}}
\end{array}\right]
$$

Step 3: Evaluate cost function value for each raindrop as follows:

$$
C_{i}=\operatorname{Cost}_{i}=f_{i}\left(x_{1}^{i}, x_{2}^{i}, \ldots, x_{N}^{i}\right), \quad i=1,2, \ldots, N_{p o p}
$$

Step 4: Define sea, rivers, and streams based on cost function values of raindrops as follows:

$$
\begin{gathered}
N_{s r}=\text { Number of River }+1 \text { (sea) } \\
N_{\text {stream }}=N_{p o p}-N_{s r}
\end{gathered}
$$

where $N_{s r}$ is the total number of and rivers and a sea.

Step 4: Define the intensity of flow for rivers and sea using Equation (12):

$$
N S_{n}=\text { round }\left\{\left|\frac{\text { Cost }_{n}}{\sum_{i=1}^{N_{s r} \text { Cost }_{i}} \mid}\right| \times N_{\text {stream }}\right\} \text { for } n=1,2, \ldots, N_{s r}
$$

Step 5: The streams flow into the rivers using Equation (13):

$$
\vec{X}_{\text {Stream }}^{i+1}=\vec{X}_{\text {Stream }}^{i}+\operatorname{rand} \times C \times\left(\vec{X}_{\text {River }}^{i}-\vec{X}_{\text {Stream }}^{i}\right)
$$

Step 6: If a stream yields a better solution than the river, the position of the stream will exchange with the river;

Step 7: Some streams can flow into the sea using Equation (14):

$$
\vec{X}_{\text {Stream }}^{i+1}=\vec{X}_{\text {Stream }}^{i}+\text { rand } \times C \times\left(\vec{X}_{\text {Sea }}^{i}-\vec{X}_{\text {Stream }}^{i}\right)
$$

Step 8: If a stream yields a better solution than the sea, the position of the stream will exchange with the sea:

Step 9: Rivers flow into the sea by equation (15):

$$
\vec{X}_{\text {River }}^{i+1}=\vec{X}_{\text {River }}^{i}+\operatorname{rand} \times C \times\left(\vec{X}_{\text {Sea }}^{i}-\vec{X}_{\text {River }}^{i}\right)
$$


Step 10: If a river yields a better solution than the sea, the position of the river will exchange with the sea;

Step 11: Check for evaporation condition:

$$
\text { if }\left\|\vec{X}_{\text {Sea }}^{i}-\vec{X}_{\text {River }}^{i}\right\| \leq d_{\max } \text { or rand }<0.1, \quad i=1,2, \ldots, N_{s r}-1
$$

Step 12: If the evaporation condition is met, the raining process will perform via equation (17):

$$
\vec{X}_{\text {Stream }}^{\text {new }}=L \vec{B}+\operatorname{rand} \times(U \vec{B}-L \vec{B})
$$

Step 13: Update $d_{\text {max }}$ using equation (18):

$$
d_{\max }^{i+1}=d_{\max }^{i}-\frac{d_{\max }^{i}}{\text { MaxIteration }}
$$

Step 14: If the stopping criteria are met, the optimization process will be terminated; if otherwise back to Step 5 .

\section{Simulation results}

To validate the performance of the proposed method using WCA, it was applied to 33node and 69-node systems. The obtained results of WCA were compared with those of other methods available in the literature. The WCA based methodology was developed by Matlab R2020a. WCA was run 30 trials independently for each test. The initial parameters of WCA $\left(N_{\text {pop }}, N_{s r}, d_{\max }\right.$, MaxIteration) were set to $30,3,1 \mathrm{E}-5$, and 100 , respectively.

\subsection{3-node EDS}

The 33-node EDS includes 33 nodes and 37 branches. There are 32 closed switches and 5 opened switches. The system data were referenced from (Baran \& Wu, 1989). Figure 1 shows the schematic diagram of the 33-node EDS. In a normal operation, switches \{3334-35-36-37\} are opened.

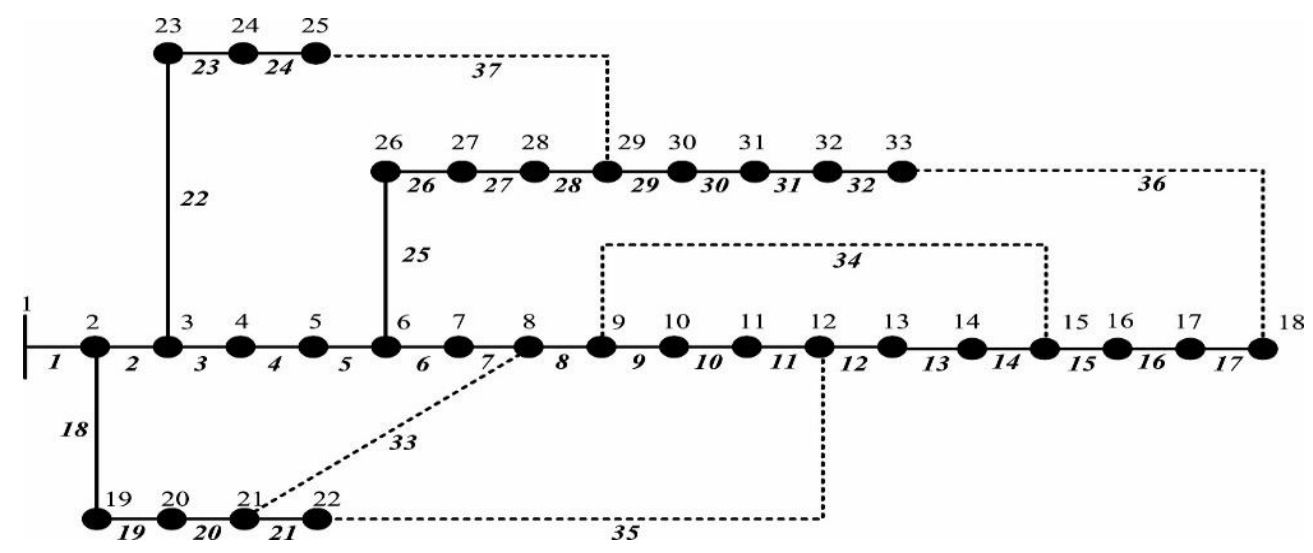

Figure 1. The 33-node EDS 
The optimal results for 33-node EDS are given in Table 1. The active power loss of the system was decreased from $202.66 \mathrm{~kW}$ to $139.5513 \mathrm{~kW}$ corresponding to a $31.14 \%$ reduction using the WCA. The active power loss value was obtained by opening the switches $\{7-9-14-32-37\}$ replacing the switches $\{33-34-35-36-37\}$. Figure 2 presents the convergence curve of WCA for 33-node EDS. Figure 3 shows the voltage of nodes in the 33-node EDS after DSR. From Figure 3, most of the voltage of nodes were improved after opening the switches $\{7-9-14-32-37\}$ and no node violates the voltage constraints. Therefore, DSR was very effective in improving the quality of the EDS. The minimal voltage amplitude was increased from 0.9131 p.u. before DSR to 0.9378 p.u. after DSR.

Moreover, the results obtained by WCA were compared to the studies in the literature in Table 1. WCA obtained similar results as recent methods such as PSO (Karimianfard \& Haghighat, 2019), uniform voltage distribution algorithm (UVDA) (Bayat, Bagheri, \& Noroozian, 2016), and salp swarm algorithm (SSA) (Sambaiah \& Jayabarathi, 2019), and better than FWA (Mohamed Imran et al., 2014) and CSA (Nguyen et al., 2016). These comparisons showed that the results obtained from the proposed WCA method were reliable.

TABLE 1. The compared results of WCA with other methods for the 33-node EDS

\begin{tabular}{|c|c|c|c|}
\hline Items & Opened switches & Power loss (kW) & $V_{\min }$ (p.u.) \\
\hline Base case & $33-34-35-36-37$ & 202.66 & 0.9131 \\
\hline The proposed WCA & $7-9-14-32-37$ & 139.5513 & 0.9378 \\
\hline FWA (Mohamed Imran et al., 2014) & $7-14-9-32-28$ & 139.98 & 0.9413 \\
\hline CSA (Nguyen et al., 2016) & $7-14-9-32-28$ & 139.98 & 0.9413 \\
\hline PSO (Karimianfard \& Haghighat, 2019) & $7-9-14-32-37$ & 139.5543 & 0.9378 \\
\hline UVDA (Bayat et al., 2016) & $7-9-14-32-37$ & 139.5543 & 0.9378 \\
\hline SSA (Sambaiah \& Jayabarathi, 2019) & $7-9-14-32-37$ & 139.5543 & 0.9378 \\
\hline
\end{tabular}

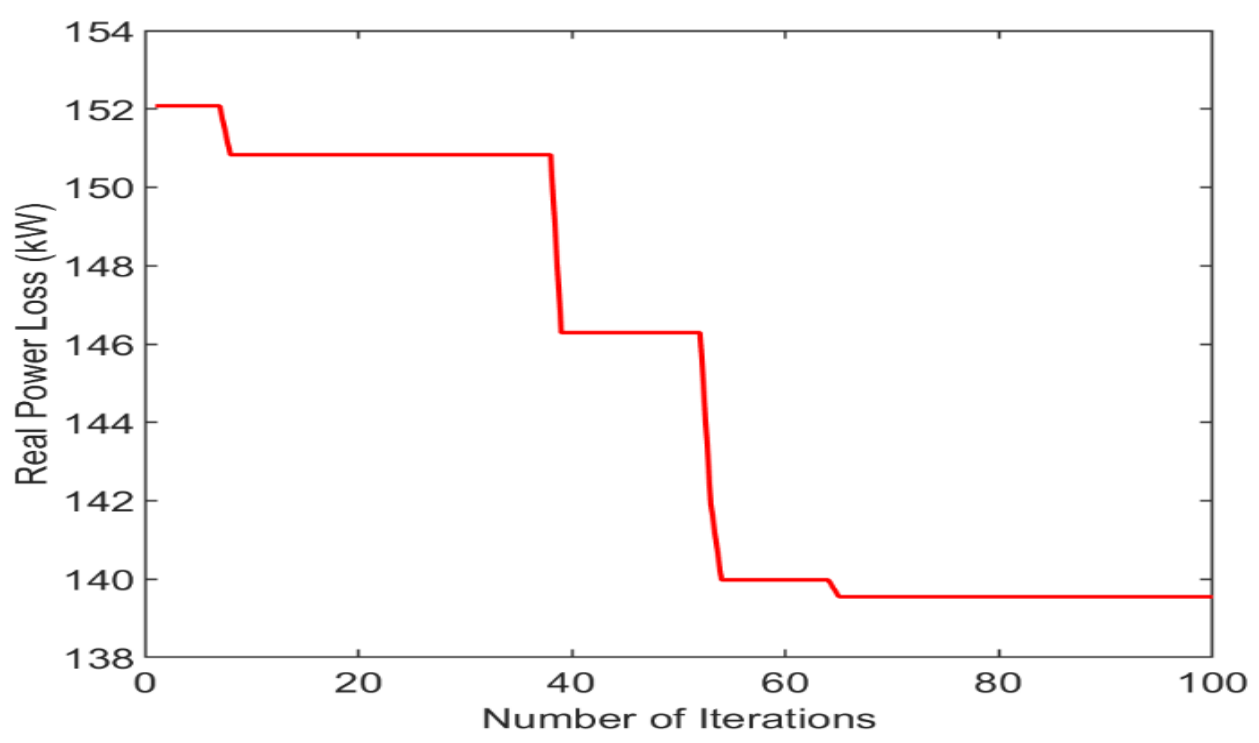

Figure 2. Convergence curve of WCA for 33-node EDS 


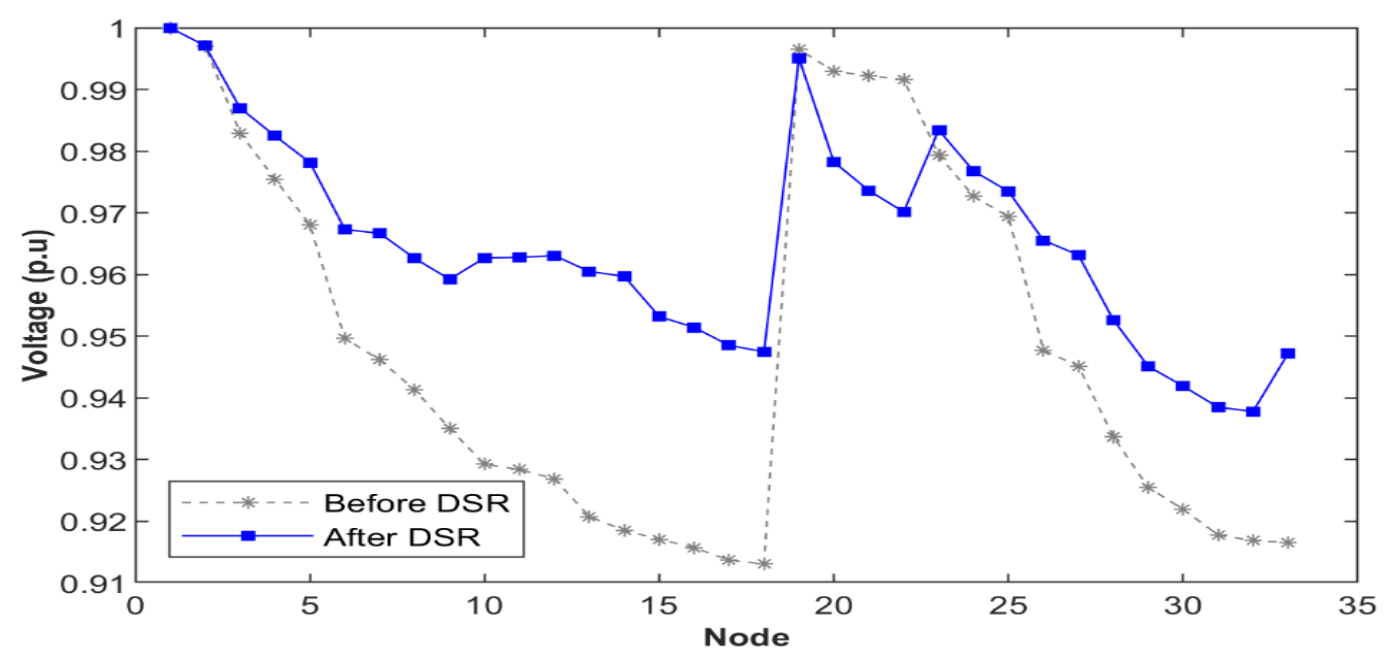

Figure 3. The node voltage amplitude of the 33-node EDS before and after DSR

\subsection{9-node EDS}

The 69-node EDS includes 69 nodes, 73 branches. There are 68 closed switches and 5 opened switches. The system data were referenced from (Baran \& Wu, 1989). Figure 4 shows the schematic diagram of the 69-node EDS. In a normal operation, switches $\{69-$ 70-71-72-73\} are opened.

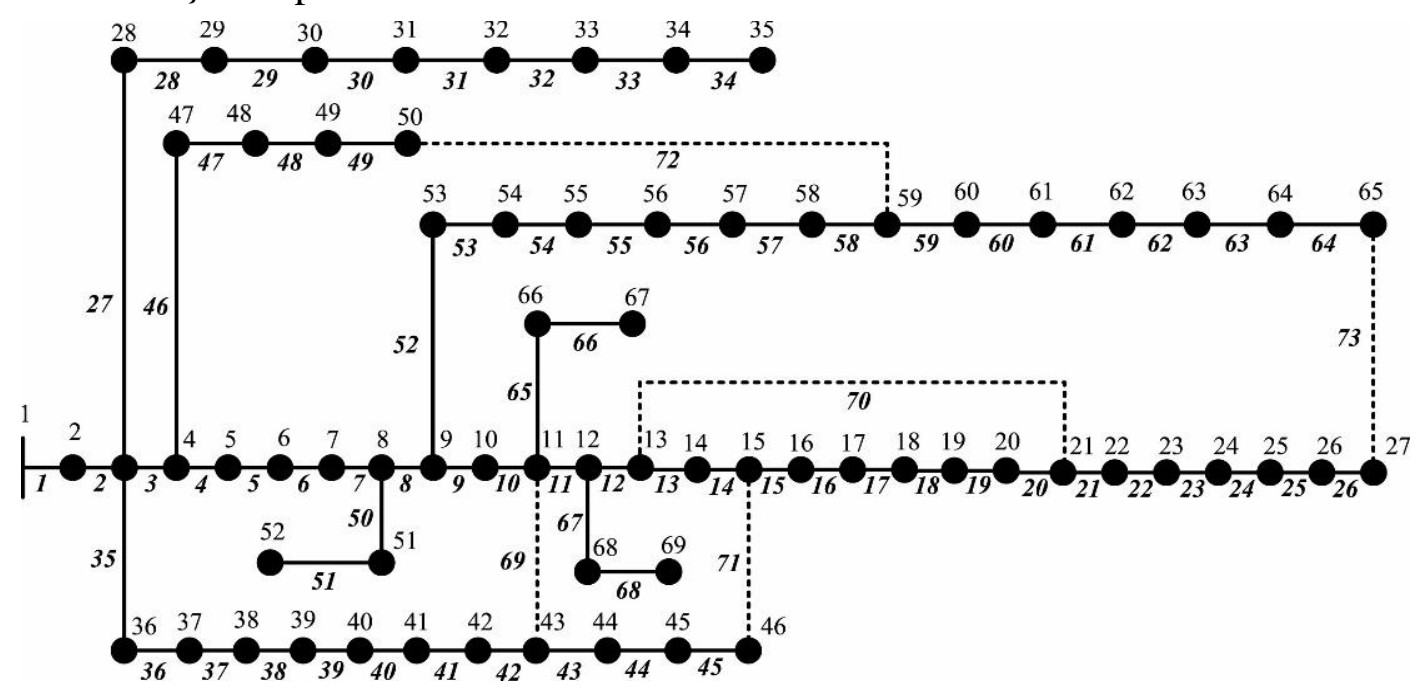

Figure 4. The 69-node EDS

The obtained results on the 69-node EDS are given in Table 2. The active power loss of the system was decreased from $224.8871 \mathrm{~kW}$ to $98.5875 \mathrm{~kW}$ corresponding to $56.16 \%$ using WCA. The active power loss value was yielded by opening the switches $\{14-57-$ 61-69-70\} replacing the switches $\{69-70-71-72-73\}$. Figure 5 presents the convergence curve of WCA for 69-node EDS. Figure 6 shows the voltage of nodes in the 69-node EDS after DSR. As can be seen in Figure 6, most of the voltage of nodes were improved after opening the switches $\{14-57-61-69-70\}$ after DSR. The minimal voltage amplitude was increased from 0.9092 p.u. before DSR to 0.9495 p.u. Hence, system performance 
was improved pointedly using WCA.

Comparison of the optimal results obtained by WCA to the studies in the literature in Table 2. The results obtained by WCA was identical to the results obtained by CSA (Nguyen et al., 2016), PSO (Karimianfard \& Haghighat, 2019), and UVDA (Bayat et al., 2016) and better than SSA (Sambaiah \& Jayabarathi, 2019). Hence, the WCA was not inferior and tended to outperform other algorithms for 69-node EDS.

TABLE 2. The compared results of WCA with other methods for the 69-node EDS

\begin{tabular}{|c|c|c|c|}
\hline Items & Opened switches & Power loss $(\mathrm{kW})$ & $V_{\min }$ (p.u.) \\
\hline Base case & $69-70-71-72-73$ & 224.8871 & 0.9092 \\
\hline The proposed WCA & $14-57-61-69-70$ & 98.5875 & 0.9495 \\
\hline CSA (Nguyen et al., 2016) & $69-70-14-57-61$ & 98.59 & 0.9495 \\
\hline PSO (Karimianfard \& Haghighat, 2019) & $14-58-61-69-70$ & 98.5875 & 0.9495 \\
\hline UVDA (Bayat et al., 2016) & $14-58-61-69-70$ & 98.5875 & 0.9495 \\
\hline SSA (Sambaiah \& Jayabarathi, 2019) & $69-14-71-61-58$ & 98.63 & 0.9492 \\
\hline
\end{tabular}

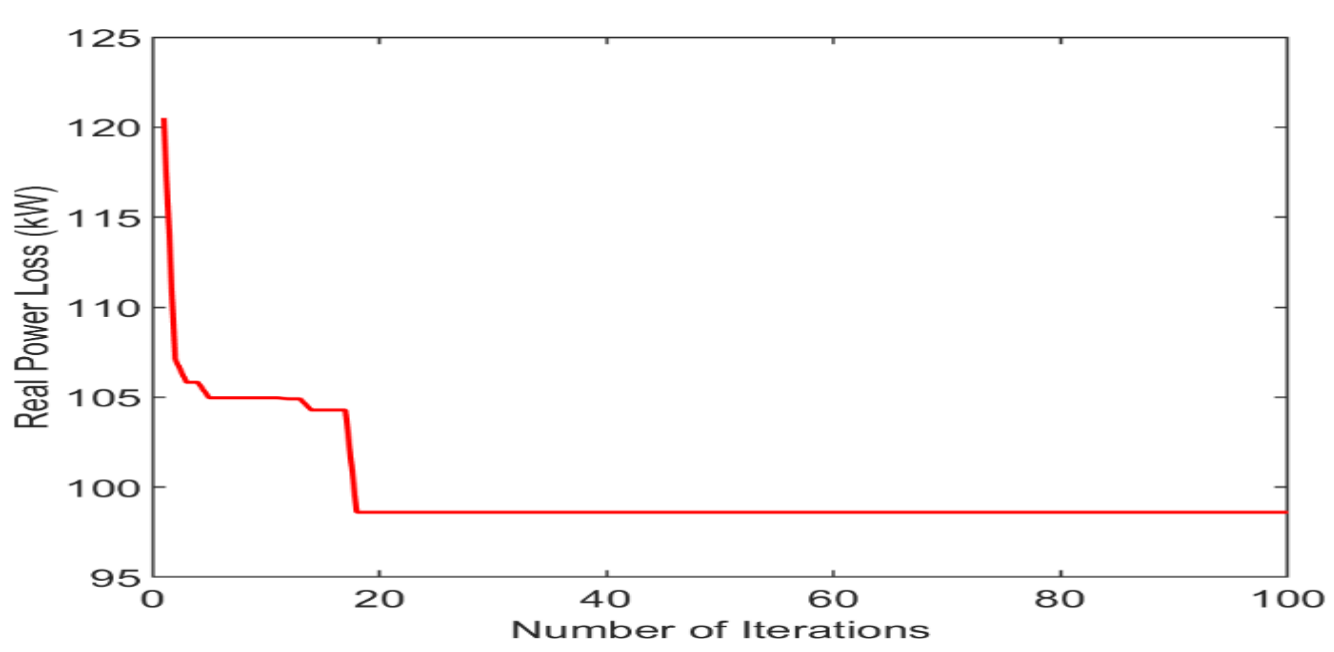

Figure 5. Convergence curve of WCA for 69-node EDS

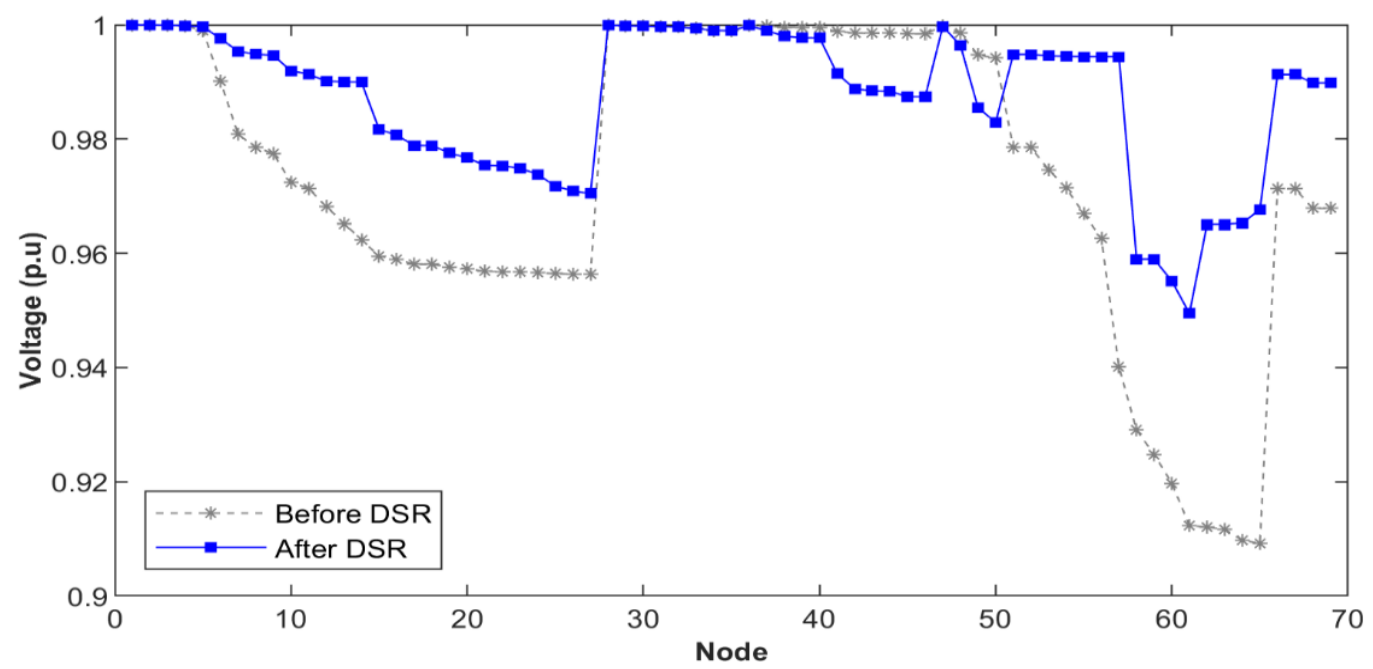

Figure 6. The node voltage amplitude of the 69-node EDS before and after DSR 


\section{Conclusion}

In this study, the WCA was successfully applied for the DSR problem. The objective is to minimize the active power loss of EDS. The effectiveness of the proposed method was evaluated on 33-node and 69-node EDS. From the optimal results, the DSR method based on WCA was effective in reducing power loss and improving the voltage profile of the system. The obtained results showed that WCA tended to outperform other methods in terms of the quality of the obtained optimal solution. Hence, WCA is the potential method for the DSR problem of reducing power loss. For further research, WCA should be implemented for other optimization problems in EDS.

\section{References}

Abdelaziz, A. Y., Mohamed, F. M., Mekhamer, S. F., \& Badr, M. A. L. (2010). Distribution system reconfiguration using a modified Tabu Search algorithm. Electric Power Systems Research, 80(8), 943-953. https://doi.org/10.1016/j.epsr.2010.01.001

Abdelaziz, A. Y., Mohammed, F. M., Mekhamer, S. F., \& Badr, M. A. L. (2009). Distribution Systems Reconfiguration using a modified particle swarm optimization algorithm. Electric Power Systems Research, 79(11), 1521-1530. https://doi.org/10.1016/j.epsr.2009.05.004

Baran, M. E., \& Wu, F. F. (1989). Network reconfiguration in distribution systems for loss reduction and load balancing. IEEE Transactions on Power Delivery, 4(2), 1401-1407. https://doi.org/10.1109/61.25627

Bayat, A., Bagheri, A., \& Noroozian, R. (2016). Optimal siting and sizing of distributed generation accompanied by reconfiguration of distribution networks for maximum loss reduction by using a new UVDA-based heuristic method. International Journal of Electrical Power \& Energy Systems, 77, 360-371. https://doi.org/10.1016/j.jjepes.2015.11.039

Biswas, P. P., Mallipeddi, R., Suganthan, P. N., \& Amaratunga, G. A. J. (2017). Optimal reconfiguration and distributed generator allocation in distribution network using an advanced adaptive differential evolution. IEEE Symposium Series on Computational Intelligence (SSCI), 1-7. https://doi.org/10.1109/SSCI.2017.8280824

Eskandar, H., Sadollah, A., Bahreininejad, A., \& Hamdi, M. (2012). Water cycle algorithm - A novel metaheuristic optimization method for solving constrained engineering optimization problems. Computers \& Structures, 110-111, 151-166. https://doi.org/10.1016/j.compstruc.2012.07.010

Karimianfard, H., \& Haghighat, H. (2019). An initial-point strategy for optimizing distribution system reconfiguration. Electric Power Systems Research, 176, 105943. https://doi.org/10.1016/j.epsr.2019.105943

Mohamed Imran, A., \& Kowsalya, M. (2014). A new power system reconfiguration scheme for power loss minimization and voltage profile enhancement using Fireworks Algorithm. International Journal of Electrical Power \& Energy Systems, 62, 312-322. https://doi.org/10.1016/j.ijepes.2014.04.034

Mohamed Imran, A., Kowsalya, M., \& Kothari, D. P. (2014). A novel integration technique for optimal network reconfiguration and distributed generation placement in power distribution networks. International Journal of Electrical Power \& Energy Systems, 63, 461-472. https://doi.org/10.1016/j.ijepes.2014.06.011

Naveen, S., Sathish Kumar, K., \& Rajalakshmi, K. (2015). Distribution system reconfiguration for loss minimization using modified bacterial foraging optimization algorithm. International Journal of Electrical Power \& Energy Systems, 69, 90-97. https://doi.org/10.1016/j.jepes.2014.12.090 
Nguyen, T. T., \& Nguyen, T. T. (2019). An improved cuckoo search algorithm for the problem of electric distribution network reconfiguration. Applied Soft Computing, 84, 105720. https://doi.org/10.1016/j.asoc.2019.105720

Nguyen, T. T., \& Truong, A. V. (2015). Distribution network reconfiguration for power loss minimization and voltage profile improvement using cuckoo search algorithm. International Journal of Electrical Power \& Energy Systems, 68, 233-242. https://doi.org/10.1016/j.ijepes.2014.12.075

Nguyen, T. T., Truong, A. V., \& Phung, T. A. (2016). A novel method based on adaptive cuckoo search for optimal network reconfiguration and distributed generation allocation in distribution network. International Journal of Electrical Power \& Energy Systems, 78, 801-815. https://doi.org/10.1016/j.ijepes.2015.12.030

Othman, A. M., El-Fergany, A. A., \& Abdelaziz, A. Y. (2015). Optimal Reconfiguration Comprising Voltage Stability Aspect Using Enhanced Binary Particle Swarm Optimization Algorithm. Electric Power Components and Systems, 43(14), 1656-1666. https://doi.org/10.1080/15325008.2015.1041623

Sambaiah, K. S., \& Jayabarathi, T. (2019). Optimal reconfiguration and renewable distributed generation allocation in electric distribution systems. International Journal of Ambient Energy, 0(0), 1-14. https://doi.org/10.1080/01430750.2019.1583604

Srinivasa Rao, R., Narasimham, S. V. L., Ramalinga Raju, M., \& Srinivasa Rao, A. (2011). Optimal Network Reconfiguration of Large-Scale Distribution System Using Harmony Search Algorithm. IEEE Transactions on Power Systems, 26(3), 1080-1088. https://doi.org/10.1109/TPWRS.2010.2076839

Zhu, J. Z. (2002). Optimal reconfiguration of electrical distribution network using the refined genetic algorithm. Electric Power Systems Research, 62(1), 37-42. https://doi.org/10.1016/S03787796(02)00041-X 